\title{
Zuiderent-Jerak Teun (2015) Situated Intervention: Sociological Experiments in Health Care. Cambridge \& London: The MIT Press. 248 pages.
}

\author{
Brit Ross Winthereik \\ IT University of Copenhagen, Denmark / brwi@itu.dk. \\ Jessica Mesman \\ Maastricht University, The Netherlands / j.mesman@maastrichtuniversity.nl. \\ Noortje Marres \\ Warwick University, UK/n.marres@warwick.ac.uk.
}

In October 2015, we got together at Linköping University in Sweden to celebrate the publication of Situated Intervention: Sociological Experiments in Health Care. Each of us - with Jessica Mesman participating via tele-conference - presented a commentary on the book, and here we present a jointly written, cooked down version of the discussion.

Situated Intervention is based on an ethnographic account of Teun Zuiderent-Jerak's experience as a researcher advising on quality improvement and cost efficiency in Dutch hospitals at the beginning of the millennium, when the Dutch government was implementing a new insurance-based healthcare paradigm. Set within this wider context, the book narrates Zuiderent-Jerak's sociologically-informed attempt to intervene in the management and doing of health care in situ. It makes an original and audacious argument that such an interventionist approach does not just have practical advantages, but presents an especially effective, experimental method of inquiry into health care.

As such, the aim of the book is also to outline a methodological strategy, one that is closely engaged with practice but at the same time expands 'the narrow definition of usefulness in scholarly work' (p. 8). This expansion happens, Zuiderent-Jerak argues, through attempts at redefining various problems and problem spaces. The problems and problem spaces dealt with in the book are those of compliance, standardization, patient safety, and health care markets, and the book explores how these were dealt with in hemophilia, hematology and oncology departments. Expanding the problem definitions associated with these topics, however, is no smooth ride, as is demonstrated throughout the book.

The book then examines the bumps on the road of situated problem-solving, showing how the constraints we run into partly derive from the fact that the issues in question are already problematized by powerful societal actors and institutions, like doctors and hospital management, in partial, interested, and forceful ways. However, Zuiderent-Jerak does not shy away from attacking established truths in academic discourse as well as professional practice about the order of things in the hospital. For example about the nurses' role, 
about methods for visualizing care paths, or about the dynamics of health care markets.

Being present in the hospital, and becoming present to the distinctive and surprising problematizations emerging in this setting is what Situated Intervention is about. It is about the consequences of being equally responsible and accountable to health care managers, clinicians, politicians, patients, nurses, academic colleagues, and scholarly (inter)disciplines. It is motivated by the ambition to find ways of attaching differently to actors in a varied and dynamic field of action, where the good and bad, the relevant and irrelevant, have not been sorted prior to the study.

\section{Situated standardization}

Situated Intervention also takes on the challenge of attempting to bypass the binary beliefs that structure many of the debates related to the improvement of health care. Indeed, the book outlines the methodological strategy of situated intervention in relation to an impressive number of dichotomies: knowing and doing; the production of knowledge and the engagement with practice; objectivism and activism; a standardized and an individualized healthcare; experimenting and intervening; universal and individual; contributing to research and practice improvement; efficiency and quality, just to name a 'few'.

Zuiderent-Jerak argues that disputes along these oppositional axes prove to be unproductive to deal with the messiness and complexities of the lived experience in daily health care practices. When oppositions like the general and the particular act as the basis for improvement measures like standardization, problems will not resolve. Therefore, a different normative approach to problems of standardization should be explored: situated standardization. In this way Zuiderent-Jerak finds a way out of a dichotomized understanding of clinical practices without closing, 'bridging' or denying the gaps between the general and the specific, the real and the ideal. Situated standardization, he argues, is instead an attempt to re-orient our relation away from 'the big gaps, towards problems. This in turn enables a mode of intervening, in which we may attempt to re-negotiate relations, including those established by and with standards. Standards, in this view, are not the foundation to which clinical realities must adjust, but resources for action, i.e. temporary outcomes of a process-driven practice in which specific issues can be made available for (sociologically) unpacking. Now standardization is no more about implementation of pre-fixed norms, but about negotiable changes and a way of doing politics, in which the researcher is deeply involved.

With the notion of situated standardization the book moves away from health care improvement as the quest for 'finding answers to pre-defined problems' to 'the articulation of new agendas' ( $p$. 158). In order to make this move Zuiderent-Jerak turns to Annemarie Mol's idea of multiple ontologies as analytical strategy to explore how different versions of 'the same' phenomena are enacted simultaneously. The simultaneity of different 'doings' of the world creates opportunities and limitations for action. By introducing the idea of multiple ontologies Zuiderent-Jerak provides us with a tool to explore the different ways in which compliance, standardization, safety or marketization arise as issues in practice. But in addition to unpacking the practices, Zuiderent-Jerak seeks to also re-pack them. Thus, the intervention is a two-step process: First, one empirically unpacks specific phenomena and issues. This can be done by experimental intervention: for example by adding a data logger to a blood cooling box and on the basis of the measurements discuss with the clinicians, who are eager to get different representations of patient worlds, what new knowledge is gained. Second, the elements that come out of this diagnostic process are conceptually, materially and organizationally re-assembled into a working configuration, based on what was already present in the situation. Thus, situated intervention is the result of a reconfiguration, not an intention set to bring in 'the new' or to 'fix' problems in the current health care system.

\section{The problem of engagement}

Situated Intervention is also about engagement. In fact, it is about engagements, as Zuiderent-Jerak presents two different forms of it and makes a passionate critique of existing approaches to both forms. The first form is about the engagement of social actors - patients, nurses, doctors - in healthcare practices. The second is about sociol- 
ogy's engagement in attempting to change the world. The book begins by reframing the problem of engagement that sociologists face. The problem of how to practice 'engaged sociology' is often misunderstood, Zuiderent-Jerak argues: it is assumed that the challenge is how to apply our knowledge, of how to do something ethical with the knowledge we have. Zuiderent-Jerak refuses this problem. Once we define our task as 'intervention,'it becomes clear that making knowledge and engaging normatively go hand in hand, he argues. This is also what the notion of the experiment teaches us: Especially when adopting an experimental approach in fieldwork settings, the experimental operates in an epistemic register as much as a normative one. In relation to intervention, consequently, the 'gap' between understanding and engaging disappears. It's an ingenious argument, among others, because it "empiricizes" the problem of engagement. When faced, for example, with widespread attempts in health care to couple the objectives of cost-saving and quality improvement, Zuiderent-Jerak asks: how do we intervene? This is an empirical question. It is a nice argument, but as Zuiderent-Jerak himself makes clear, there are issues...

For example, isn't there something distinctive about the capacities of social researchers, theorists and scholars for intervention? By giving up engagement, how can we continue to practice sociology, intervene sociologically? ZuiderentJerak included the term sociology in his title, something that is somewhat surprising for a book informed by actor-network theory (which after all is famous for its call to 'move beyond' sociology's notion of a society that can be intervened in. Yet, Zuiderent-Jerak nicely praises sociology as a distinctive form of 'making trouble', though he also notes that its condition of success is the reconfiguration of the problem space. (This gets potentially tricky, as there are now two objects of problematization in play: the researcher's engagement is 'problematic', but then there are also the problems of healthcare. But let's leave this aside for the moment.)

However, what is also striking about ZuiderentJerak's book is his seeming lack of concern about securing his identity as a sociologist. He is not afraid to adopt un-sociological vocabulary:
Many of the book's most interesting problems are formulated in the vocabulary of health care management. For example, how to improve quality and save cost? In Zuiderent-Jerak's mode of intervention, these concerns become the sociologist's, and it thereby blurs the boundaries between health care management's concerns and sociological ones. Zuiderent-Jerak argues that sociological problems may become tractable in this way.

However, this raises the question: what about tensions? In doing both sociology and healthcare management at the same time, is Zuiderent-Jerak not presuming a win-win logic? A significant merit of the book is that it explicitly recognizes this challenge. It describes how in pursuing new ways of coupling quality and cost, this was not the outcome: Logic concerned with price ended up trumping quality. It was win-loose. In addressing this, Zuiderent-Jerak argues that we are dependent on wider ecologies of knowing and doing. He argues that we must recognize the need for 'artful contamination' between sociology and healthcare practices for intervention to stand a chance. But, how does intervention open up such a space for contamination, a space for encounter between different competences?

Here, too, Zuiderent-Jerak has an answer: situated intervention is intervention situated in issues. When problems emerge, an in-between space opens up, one where different actors (say nurses and doctors) are brought into relation. This we find terribly interesting. After all, situatedness used to be about situating stuff in specific situation at specific sites, but for Zuiderent-Jerak intervention is a way of getting involved in issues (i.e. the quality of care), and he proposes to include patient-centred care in issues. Even flowcharts, in his account, should be acknowledged as devices for articulating issues.

A further question emerges from this: How is intervention capable of opening a space of the in-between? Wasn't the argument that intervention does away with 'gaps? Here we should consider how, for Zuiderent-Jerak, intervention is also an alternative to participation. For him, participation is overrated, it is often sentimental (not based on inquiry), and risks to obscure power dynamics. He makes a relevant argument for 
intervention - as distinct from participation - on this point too. Intervention enables a focus on the patient-centred organisation of healthcare, something which Zuiderent-Jerak opposes to a more myopic focus on directly involving people, which he argues a participatory agenda often comes down to in practice. Zuiderent-Jerak's argues that patients are served better by a good flowchart than by doctors with limitless time to listen to them.

However, in advocating a shift from participation to intervention Zuiderent-Jerak runs the risk of closing down the space of the both-or and the in-between. Zuiderent-Jerak provokes us: Participation is sentimental, critique from an external position is 'easy.' But isn't participation something that brings about the in-between and connects relative strangers? Is not critique from a distance sometimes necessary for destabilizing normative complacency, and indeed for enabling precisely the unsettlement of established problem definitions, and the expansion of problem spaces, which Zuiderent-Jerak argues are so much needed? Why this need to reject participation and critique?

That said, so much is amazing about Situated Intervention. For example its definition of norma- tivity: 'The capacity to respond to variability' ( $p$. 190-92). There is an experimental ethos at heart of this definition, of trying things out, of finding out, of changing one's mind. But seeing variability among responses requires the multiplication of standpoints and identities. Zuiderent-Jerak's book takes us along on a passage through standpoints of patients, nurses, doctors, insurers, sociologists. Yet, he also shows how an interventionist sociology is able to multiply the identities of its allies, nurses for example. Health care organizations, from the perspective of the book, now become experimental, making experimenting interventions themselves all the time. This raises the question of how to mobilize this resource, this capacity to respond to variability? As ZuiderentJerak states on the book's last page social scientists can learn from this capacity and accept our normativity as embedded and enacted in practice. But how to take on the responsibility that experimentation in situ confers on experimenters? As Zuiderent-Jerak notes situated experiments inevitably operate in a normative register and expand the capacities for problem articulation and intervention not just in, but also beyond, situations. 